\title{
Fertilité et cancer du testicule. Impact des traitements.
}

\author{
Eric HUYGHE 1,2, Myriam DAUDIN 2, Louis BUJAN 2, Michel SOULIE 1 \\ Pierre PLANTE 1, Patrick Fernand THONNEAU 2 \\ ${ }^{1}$ Service d'Urologie Andrologie, CHU Rangueil, Toulouse. \\ ${ }^{2}$ Groupe de Recherche sur la fertilité humaine, CHU La Grave, Toulouse.
}

\section{RESUME}

Objectifs : Etudier la fertilité des patients atteints de cancer du testicule.

Population et Méthode : une série consécutive de 489 hommes traités pour une tumeur germinale du testicule au niveau de la région Midi-Pyrénées, entre 1978 et 1998, a été explorée du point de vue de la fertilité, grâce à un questionnaire adressé aux patients par courrier. Un taux de participation de $95 \%$ a été obtenu.

Résultats : L'information concernant la fertilité a été obtenue pour $\mathbf{4 4 6}$ hommes. Avant le cancer du testicule, 90,1\% des patients qui avaient cherché à avoir des enfants y étaient parvenus. Après traitement du cancer, seulement $61,8 \%$ des hommes parvenaient à avoir un enfant. La radiothérapie et la chirurgie des masses résiduelles apparaissent comme plus délétères pour la fertilité que la chimiothérapie de type BEP.

Conclusion : La population des hommes traités pour un cancer du testicule est à risque d'infertilité. La fécondabilité de ces patients diminue d'environ $30 \%$ après traitement.

Mots-clés : cancer du testicule, fertilité, chimiothérapie, radiothérapie

\section{INTRODUCTION}

La fertilité est l'une des préoccupations majeures des hommes atteints de cancer du testicule [5]. Cette pathologie touche des hommes jeunes (première cause de cancer chez les hommes entre 15 et 34 ans) [8], à un âge où beaucoup n'ont pas encore eu d'enfant. Parmi ceux-ci, $76 \%$ ont un projet de paternité pour le futur [18]. Les chimiothérapies et radiothérapies ont amélioré considérablement le pronostic des patients atteints de cancer du testicule [7], mais ils entraînent une atteinte de la spermatogenèse sévère et pouvant être définitive $[4,9,10,16]$. Nous avons exploré le statut de fertilité après traitement du cancer du testicule en conduisant une large étude rétrospective dans la région Midi-Pyrénées.

\section{MATERIEL ET METHODES}

En 1999, nous avons repris la série consécutive des 506 patients chez lesquels une tumeur du testicule avait été diagnostiquée entre 1979 et 1999 au niveau des structures de soins de la région Midi-Pyrénées (services d'urologie et d'oncologie publics et privés, CECOS Midi-Pyrénées).

Nous avons décidé de limiter l'étude aux tumeurs germinales du testicule. Nous avons exclu les patients porteurs d'un lymphome $(\mathrm{n}=17)$, d'une tumeur à cellules de Leydig $(n=17)$, et d'une tumeur à cellules de Sertoli $(n=1)$. Nous

Correspondance :

Dr Patrick Thonneau - Groupe de Recherche en Fertilité Humaine - Service d'Urologie-Andrologie. Hôpital La Grave. TSA 60033 - 31059 Toulouse Cedex 9. France -

Tel 05.61.77.78.41 - Fax 05.61.77.78.43 -

Email thonneau.p@chu-toulouse.fr 
avons également exclu deux patients pour lesquels nous n'avions pas de diagnostic histologique fiable.

Les patients ont ensuite été classés en fonction de leur histologie, de leur stade clinique, et du traitement administré. Cinq groupes ont été définis : groupe $1(n=18)$ comprenant les patients présentant un stade I, traités par orchidectomie seule ou par orchidectomie et curage ganglionnaire rétropéritonéal. Groupe $2(n=112)$ comprenant les patients présentant une tumeur germinale non séminomateuse de stade I, IIa et IIb, traités par orchidectomie et chimiothérapie à base de platine (PVB, BEP, EP). Groupe $3(n=158)$ comprenant les patients ayant un séminome de stade I, IIa, et IIb traités par orchidectomie et radiothérapie (irradiation de 25 à 35 Gy). Groupe $4(n=87)$ comprenant les patients en rechute, ou présentant une tumeur de stade avancé (stades IIc et III) traités par orchidectomie et chimiothérapie intensive avec ou sans radiothérapie. Groupe $5(\mathrm{n}=44)$ comprenant les patients ayant nécessité une chirurgie des masses résiduelles après chimiothérapie.

L'exploration de la fertilité a été réalisée à l'aide d'un questionnaire conçu au sein du Groupe de Recherche sur la Fertilité Humaine, permettant de connaître avec précision l'ensemble de l'histoire reproductive de ces patients. Ce questionnaire a été adressé aux patients par voie postale, accompagné d'une lettre d'information et d'une notice explicative. Il était articulé en deux volets : la fertilité avant la tumeur, et la fertilité après les traitements.

Après deux relances par courrier, et une relance téléphonique, nous avons obtenu un taux de participation de $95 \%$.

L'analyse statistique a été réalisée avec le logiciel STATA, en prenant le degré de signification à 0,05 . Les comparaisons de sous-groupes ont été réalisées avec le test du Chi 2. Les différents facteurs de risque d'infertilité ont été analysés par régression logistique.

\section{RESULTATS}

Parmi les 489 patients ayant une tumeur germinale, nous avons obtenu l'information concernant leur traitement chez $453(96,6 \%)$ et l'information concernant la fertilité chez $446(95,1 \%)$ d'entre eux.

Notre population comportait 215 séminomes ( $47 \%$ ), et 238 tumeurs germinales non séminomateuses $(53 \%)$. Parmi ces dernières, on dénombrait $2,4 \%$ de tératomes, $16,8 \%$ de carcinomes embryonnaires, $1,3 \%$ de choriocarcinomes purs, $0,9 \%$ de tumeurs vitellines, et $31,6 \%$ de tumeurs mixtes.

L'âge moyen était de 26 ans (14 - 64) chez les patients présentant une tumeur germinale non séminomateuse, et de 34 ans (15 - 73) chez les patients présentant un séminome. Nous avions une information d'antécédent de cryptorchidie chez 56 patients $(11,9 \%)$.
Le suivi était de 83 mois (36 à 316). Soixante-six patients ont présenté une rechute et 35 sont décédés de leur cancer (taux de mortalité liée au cancer $=7,7 \%$ ).

Avant le cancer, la proportion d'hommes qui avaient cherché à avoir des enfants était de 242 sur 446, et celle des hommes qui avaient eu un enfant était de 218 (90,1\%). Après le traitement, sur 170 qui avaient cherché à avoir un enfant, seuls 105 avaient réussi $(61,8 \%)$. Le délai moyen entre la fin des traitements et la naissance du premier enfant suivant le traitement était de 4,8 ans $( \pm 3)$.

Le recours aux paillettes de sperme mises en banque a permis à 5 couples d'avoir un enfant.

Les principaux facteurs de risque d'infertilité sont le fait d'avoir une histoire de cryptorchidie, et d'être oligosperme au moment de la conservation de sperme. Concernant l'impact des traitements, le risque d'infertilité est majoré en cas de chirurgie des masses résiduelles. Par ailleurs, le risque d'infécondité est significativement plus important après radiothérapie qu'après chimiothérapie de type BEP.

\section{DISCUSSION}

Le résultat principal de notre étude était d'établir que 105 hommes, parmi les 170 hommes ayant cherché à avoir un enfant après traitement, étaient parvenus à concevoir, soit une proportion d'hommes fertiles de $61 \%$. Nos résultats sont assez voisins de ceux de Hartmann [14] qui retrouvait que $53 \%$ des couples (21 sur 40 ) qui essayaient d'avoir une grossesse après cancer du testicule y parvenaient. Cependant, sa population comportait $25 \%$ de patients ayant eu une chirurgie des masses résiduelles, traitement qui est bien connu pour entraîner des troubles mécaniques pouvant gréver négativement leur fertilité. Bohlen [6] retrouvait 11 hommes fertiles sur $16(68 \%)$ et Herr [15], 41 fertiles sur 63 hommes $(65 \%)$. Cependant ces deux dernières études s'adressaient à des patients ayant une maladie localisée de stade I, soumis à des traitements peu agressifs sur la fertilité. Deux autres études retrouvaient des proportions plus faible d'hommes fertiles après traitement : 11 sur 39 (28\%) pour Arai [2], et 16 sur 41 (39\%) pour Hansen [12]. On peut remarquer que ces deux études incluaient des patients ayant un recul de moins d'un an par rapport à la fin des traitements. Il semble bien que la proportion d'hommes parvenant à concevoir après traitement soit d'environ $60 \%$.

Concernant l'effet des traitements du cancer du testicule sur la fertilité, les résultats dont nous disposons dans la littérature étudient pour la plupart l'évolution de leurs paramètres spermatiques après traitement.

Fossa [11] a rapporté que sur 53 patients qui étaient fertiles avant radiothérapie, 41 étaient parvenus à avoir des enfants après radiothérapie infra diaphragmatique. Cependant, Hansen [13] observait que chez 51 patients avant radiothé- 
rapie, $26(51 \%)$ avaient une numération spermatique basse. Deux ans après traitement, tous les patients avaient une numération spermatique basse, et cette oligospermie persistait huit ans après traitement chez 14 des 17 patients suivis (82\%).

Nijman [17] a étudié les paramètres du sperme de 25 patients traités par quatre cycles de PVB. Avant chimiothérapie, 18 patients avaient des concentrations spermatiques inférieures à 20 millions par $\mathrm{ml}$, et un patient $(4 \%)$ était azoosperme. Un an après la fin des traitements, 21 patients avaient des concentrations spermatiques inférieures à 20 millions par $\mathrm{ml}$, parmi lesquels $12(40 \%)$ étaient azoospermes. Deux ans après traitement, 12 des 25 patients avaient des concentrations spermatiques inférieures à 20 millions par $\mathrm{ml}$, et sept patients $(28 \%)$ restaient azoospermes.

A côté des études spermiologiques, des études rapportant les événements reproductifs de ces patients après traitements, donnaient des résultats discordants. Une étude portant sur 85 survivants du cancer du testicule retrouvait une proportion d'hommes fertiles très basse chez les patients traités par chimiothérapie et curage ganglionnaire rétropéritonéal ( 2 sur 19 patients), et par radiothérapie ( 4 sur 40 patients). Dans le groupe des patients traités par orchidectomie seule (surveillance exclusive), 4 des 9 patients étaient parvenus à avoir des enfants. Cependant aucune différence significative ne pouvait être mise en évidence compte tenu de la faiblesse des effectifs [2]. Hansen, en 1991, ne dégagea pas de différence de fécondité entre les hommes ayant reçu un traitement chimiothérapique ou radiothérapique et ceux n'en ayant pas reçu [12]. En Suisse, Bohlen concluait à une absence d'effet de la chimiothérapie adjuvante (2 cures de BEP) sur la fertilité [6]. Hartmann identifiait le groupe des patients ayant eu une chirurgie des masses résiduelles comme groupe à risque majoré d'infertilité [14]. Notre étude est la première à montrer que le traitement radiothérapique administré dans le cadre du cancer du testicule retentit de manière plus sévère sur la fertilité que la chimiothérapie de type BEP.

En conclusion, cette étude réalisée sur plus de $\mathbf{5 0 0}$ patients, avec un taux de participation de $95 \%$, démontre qu'après traitement du cancer du testicule, la fécondabilité diminue d'environ $30 \%$; ce travail identifie aussi les patients ayant reçu une radiothérapie, et ceux ayant subi une chirurgie des masses résiduelles comme des populations à risque majoré d'infertilité. Cette étude réaffirme l'importance de la conservation du sperme avant l'initiation des traitements, et le rôle des $\mathrm{C}$ E O O $[1,3]$.

\section{REFERENCES}

1 AGARWAL A. : Semen banking in patients with cancer : 20-year experience. Int. J. Androl., 2000, 23 (suppl.2) : 16-19.

2 ARAI Y., KAWAKITA M., OKADA Y., YOSHIDA O. : Sexuality and fertility in long-term survivors of testicular cancer. J. Clin. Oncol., 1997, $15:$ 1444-1448.

3 AUGER J., KUNSTMANN J.M., CZYGLICK F., JOUANNET P. : Prévention des risques d'infertilité liés aux traitements antitumoraux dans le cancer du testicule. Andrologie, 2000, 2 : 194 202.

4 BACHAUD J.M., ALZIEU C., BUJAN L. : Effets de la radiothérapie sur la fonction testiculaire de l'adulte. Andrologie, 1995 $5: 476-485$.

5 BERTHELSEN J. G. : Testicular cancer and fertility. Int. J. Androl., 1987, $10: 371-380$.

6 BOHLEN D., BURKHARD F.C., MILLS R., SONNTAG R.W., STUDER U. E. : Fertility and sexual function following orchiectomy and 2 cycles of chemotherapy for stage I high risk nonseminomatous germ cell cancer. J. Urol., 2001, $165: 441-444$.

7 BOSL G.J., MOTZER R.J. : Testicular germ-cell cancer. N. Engl. J. Med., 1997, $337:$ 242-253.

8 BUETOW S.A. : Epidemiology of testicular cancer. Epidemiol. Rev., 1995, $17: 433-449$.

9 CHEVREAU C., HUGUET F. : Chimiothérapie anticancéreuse et fertilité. Andrologie, 1995, $5: 458-464$.

10 COSTABILE R.A. : The effects of cancer and cancer therapy on male reproductive function. J. Urol., 1993, $149: 1327-1330$.

11 FOSSA S.D., AASS N., KAALHUS O. : Long-term morbidity after infradiaphragmatic radiotherapy in young men with testicular cancer. Cancer, 1989, $64:$ 404-408.

12 HANSEN P.V., GLAVIND K., PANDURO J., PEDERSEN M. : Paternity in patients with testicular germ cell cancer : pretreatment and post-treatment findings. Eur. J. Cancer, 1991, 27 : 13851389.

13 HANSEN P.V., TRYKKER H., SVENNEKJAER I.L., HVOLBY J. : Long-term recovery of spermatogenesis after radiotherapy in patients with testicular cancer. Radiother. Oncol,, 1990, 18 : 117125.

14 HARTMANN J.T., ALBRECHT C., SCHMOLL H.J., et al. : Long-term effects on sexual function and fertility after treatment of testicular cancer. Br. J. Cancer, 1999, $80: 801-807$.

15 HERR H.W., BAR-CHAMA N., O'SULLIVAN M., SOGANI P.C. : Paternity in men with stage I testis tumors on surveillance. J. Clin. Oncol., 1998, $16: 733-734$.

16 HOWELL S., SHALET S. : Gonadal damage from chemotherapy and radiotherapy. Endocrinol. Metab. Clin. North Am., 1998, 27 : 927-943.

17 NIJMAN J.M., SCHRAFFORDT KOOPS H., KREMER J., SLEIJFER D.T. : Gonadal function after surgery and chemotherapy in men with stage $I I$ and III nonseminomatous testicular tumors. J. Clin. Oncol., 1987, 5 : 651-656.

18 SCHOVER L.R., RYBICKI L.A., MARTIN B.A., BRINGELSEN K.A. : Having children after cancer. A pilot survey of survivors' attitudes and experiences. Cancer, 1999, 86 : 697-709. 


\section{ABSTRACT \\ Fertility and testicular cancer. \\ Impact of treatments. \\ Eric HUYGHE, Myriam DAUDIN, Louis BUJAN, Michel SOULIE \\ Pierre PLANTE, Patrick Fernand THONNEAU}

Objectives: To study the fertility of patients with testicular cancer.

Population and Method: The fertility of a consecutive series of $\mathbf{4 8 9}$ men treated for germ cell tumour of the testis in the Midi-Pyrénées region, between 1978 and 1998, was investigated by means of a questionnaire sent by mail to the patients. A participation rate of $95 \%$ was obtained.

Results: Information concerning fertility was obtained for 446 men. $90.1 \%$ of patients who had tried to have children prior to their testicular cancer had succeeded, but only $61.8 \%$ of men were able to have a child after treatment of their cancer. Radiotherapy and surgery of residual masses appear to be more harmful to fertility than BOE chemotherapy.

Conclusion: The population of men treated for testicular cancer present a high risk of infertility, as the fertility of these men decreases by about $30 \%$ after treatment.

Key Words : testis cancer, fecundity, chemotherapy, radiotherapy 